UIUC-P-95-07-050

\title{
Transport through Dirty Luttinger Liquids Connected to Reservoirs
}

\author{
Dmitrii L. Maslov \\ Department of Physics and Materials Research Laboratory \\ University of Illinois at Urbana-Champaign, Urbana, IL 61801, USA; \\ and Institute for Microelectronics Technology, Academy of Sciences of Russia \\ Chernogolovka, 142432 Russia
}

\begin{abstract}
It is shown that the conductance of a weakly disordered Luttinger-liquid quantum wire connected to non-interacting leads is affected by electron-electron interactions in the wire. This is in contrast to the case of a perfect wire the conductance of which is given by $e^{2} / h$ regardless of interactions in the wire. The disorder-induced correction to the conductance scales with temperature and/or the wire length, the scaling exponent being determined only by the interaction strength in the wire. These results explain recent experiments on quasi-ballistic GaAs quantum wires.

PACS numbers: 72.10.Bg, 73.20.Dx
\end{abstract}

Typeset Using REVTEX 
Transport through quantum wires is commonly believed to be strongly affected by electron-electron interactions. In particular, if only one channel of transverse quantization is open and the electrons are in the Luttinger liquid state [1], the conductance of a perfect wire is expected to be $K e^{2} / h$ [2 [5], where the parameter $K$ characterizes the sign and the strength of the interactions: $K<1$ for repulsion; $K>1$ for attraction; $K=1$ in the absence of interactions. Moreover, in the case of repulsion, disorder in the wire is predicted to suppress the conductance much more strongly than in the non-interacting case [2] [7. This suppression comes about via temperature- and/or length-dependent corrections to the conductance of a perfect wire that diverge in the limit $T \rightarrow 0$ or $L \rightarrow \infty$ even for an arbitrarily weak disorder, indicating the tendency to an insulating behavior. The exponents of the $T$ - and $L$ - scalings are also determined by the parameter $K$. This has to be contrasted with the non-interacting theory, which predicts $T$-independent conductance (at least for $T$ smaller than the interband energy spacing). The physical explanation of the interaction-induced breakdown of conduction [4] is that the $2 k_{F}$ backscattering due to impurities stimulates the divergence of charge-density-wave fluctuations with the period $2 \pi / 2 k_{F}$. With all these dramatic differences between the interacting and the non-interacting theories, an experiment seems capable of discriminating between the two.

At the first sight, a recent experiment on high-mobility GaAs quantum wires [8] has given support to neither theory, however. Indeed, at higher temperatures $(T>1.2 \mathrm{~K})$ the observed value of the conductance was $e^{2} / h$ with a high accuracy, which seems to support the non-interacting theory. On the other hand, reduction of the conductance was observed at lower $T$, and by fitting the observed $T$-dependence of the conductance into the interacting theory [5] the authors of Ref. [8] obtained a value of $K \approx 0.7$. This seems to support the interacting theory, but also implies that at higher $T$, where the disorder-induced correction is small, the reduction of the conductance should have been of the order of $30 \%$, which clearly contradicts to the data.

A partial resolution to this paradox has been given in two recent papers [9, 10, in which it was emphasized that the model of a homogeneous Luttinger liquid leading to the result 
$K e^{2} / h$ of Refs. [2-5] is not adequate to a typical experimental situation. Indeed, a narrow wire is always connected to wide electron reservoirs by the conducting leads. As the leads and the reservoirs are necessarily not one-dimensional, the electrons there form not a Luttinger liquid but rather a Fermi liquid which, for the present purposes, can be considered as a non-interacting Fermi-gas. Consequently, the effective one-dimensional model considered in Refs. [9, 10] was that of an inhomogeneous Luttinger liquid with the interaction strength varying from some non-zero value in the central part of the system (the "wire") to zero in the outer parts (the "leads"). It has been shown that in the absence of disorder the conductance of such a system is given by $e^{2} / h$ regardless of the interactions in the wire. This explains the absence of the conductance-renormalization at higher $T$ observed in Ref. [8]. It must be added that remarks supporting this result were made earlier by Kane and Fisher [3] and by Matveev and Glazman 11$]$.

The question that still remains open is whether the experimentally observed $T$ dependence of the conductance is a signature of a dirty Luttinger-liquid state in the wire itself or is a reflection of the presence of the leads as well. In this paper, I show that the $T$ and $L$-dependent corrections to the dc conductance of a weakly disordered wire are determined entirely by the interactions in the wire, and are not affected by the presence of the non-interacting leads. This result, taken together with the results of Refs. [9, 10], suggests that the experimental observations of Ref. [8] can be seriously considered as an indication of the Luttinger-liquid state in GaAs quantum wires.

The result announced above can be entirely anticipated from the following simple physical picture. Consider a weakly disordered wire containing a Luttinger liquid and adiabatically connected to the Fermi-liquid leads. In the absence of disorder, the finite resistance of a perfect wire $\left(=h / e^{2}\right)$ is entirely due to contact resistance [12 15]: some of the electrons coming from the wide leads are reflected as the channel becomes narrower. This reflection takes place outside the wire, where the electrons are in the Fermi-liquid state, therefore, the contact resistance is not affected by the interactions in the wire. Weak disorder in the wire gives rise to an additional contribution to the resistance. This contribution is determined 
by the scattering in the wire, where electrons are in the Luttinger-liquid state, and therefore this contribution has features typical of a Luttinger liquid but not of a Fermi liquid.

As in Refs. [9],10], I consider an infinite Luttinger liquid separated into three regions: the wire $(-L / 2<x<L / 2)$ and the leads $(x \geq L / 2)$. The interaction parameter $K$ changes abruptly from the value $K_{\mathrm{W}}$ in the wire to the value $K_{\mathrm{L}}$ in the leads. Having in mind the experimental system studied in Ref. [8], I shall focus on the case of repulsive interaction in the wire (i.e., $K_{\mathrm{W}} \leq 1$ ) and put $K_{\mathrm{L}}=1$ at the end of the calculation. The length $L$ corresponds to the length of that segment of the original system where the applied electrostatic potential varies most appreciably. Consequently, the electric field is assumed to be zero for $|x| \geq L / 2$. I also assume that a random potential $V(x)$ is present only in the wire, i.e., for $|x|<L / 2$, and is weak enough to be treated via perturbation theory [16]. The current $I=e j$ is related to the electric field by

$$
I(x, t)=\int_{-L / 2}^{L / 2} d x^{\prime} \int \frac{d \omega}{2 \pi} e^{-i \omega t} \sigma_{\omega}\left(x, x^{\prime}\right) \bar{E}_{\omega}\left(x^{\prime}\right),
$$

where $\bar{E}_{\omega}(x)$ is temporal Fourier component of the electric field at point $x$ and $\sigma_{\omega}\left(x, x^{\prime}\right)$ is the non-local ac conductivity, which is given by the Kubo formula [18]

$$
\sigma_{\omega}\left(x, x^{\prime}\right)=\left.\frac{i e^{2}}{\omega} \int_{0}^{\beta}\left\langle T_{\tau}^{*} j(x, \tau) j\left(x^{\prime}, 0\right)\right\rangle e^{-i \bar{\omega} \tau}\right|_{\bar{\omega}=i \omega-\epsilon} \cdot
$$

(I put $\hbar=1$ ). In the bosonized form, the particle-number current is given by $j=-i \partial_{\tau} \phi / \sqrt{\pi}$ and Eq. (2) reduces to 18

$$
\begin{aligned}
\sigma_{\omega}\left(x, x^{\prime}\right) & =\left.e^{2} \frac{i \bar{\omega}^{2}}{\pi \omega} G_{\bar{\omega}}\left(x, x^{\prime}\right)\right|_{\bar{\omega} \rightarrow i \omega-\epsilon}, \\
G_{\bar{\omega}}\left(x, x^{\prime}\right) & =\int_{0}^{\beta} d \tau\left\langle T_{\tau}^{*} \phi(x, \tau) \phi\left(x^{\prime}, 0\right)\right\rangle e^{-i \bar{\omega} \tau},
\end{aligned}
$$

where $G_{\bar{\omega}}$ is the propagator of the boson field $\phi$. The (Euclidean) action of the spinless Luttinger liquid is given by $S=S_{0}+S_{\mathrm{i}}$, where

$$
S_{0}=\iint_{0}^{\beta} d x d \tau \frac{1}{2 K(x)}\left\{\frac{1}{v(x)}\left(\partial_{\tau} \phi\right)^{2}+v(x)\left(\partial_{x} \phi\right)^{2}\right\},
$$

$v(x)$ is the density-wave velocity and $S_{\mathrm{i}}$, which describes backscattering due to disorder, is given by 


$$
S_{\mathrm{i}}=\frac{2}{a} \int d x \int_{0}^{\beta} d \tau V(x) \cos \left(2 k_{F} x+2 \sqrt{\pi} \phi\right)
$$

in which $a$ is the microscopic length cut-off. In the homogeneous model [2,5], $\sigma_{\omega}$ in the presence disorder was found by using the Luther-Peshel formula [19], which relates the effective mean free time to the $2 k_{F}$ component of the density-density correlator. In the inhomogeneous situation, the meaning of the mean free time is unclear, and I will determine the disorder-induced corrections to $\sigma_{\omega}$ directly from the Kubo formula (2) via perturbation theory in $S_{\mathrm{i}}$. In what follows, I will consider only the conductance averaged over the ensemble of disorder realizations. Assuming that $\overline{V(x)}=0$, where $\cdots$ stands for the ensemble averaging, the first non-vanishing correction to the ensemble-averaged propagator is given by

$$
\begin{aligned}
& \overline{\delta G\left(X, X^{\prime}\right)}=\frac{1}{a^{2}} \int d X_{1} d X_{2} \overline{V\left(x_{1}\right) V\left(x_{2}\right)} \cos \left(2 k_{\mathrm{F}}\left(x_{1}-x_{2}\right)\right) \\
& \left\{\left\langle\phi(X) \phi\left(X^{\prime}\right) Q\left(X_{1}, X_{2}\right)\right\rangle_{0}-\left\langle\phi(X) \phi\left(X^{\prime}\right)\right\rangle_{0}\left\langle Q\left(X_{1}, X_{2}\right)\right\rangle_{0}\right\}
\end{aligned}
$$

where $X=\{x, \tau\},\langle\ldots\rangle_{0}$ stands for averaging over Gaussian fluctuations of $\phi$ with the weight $S_{0}$, and

$$
Q\left(X_{1}, X_{2}\right) \equiv e^{i 2 \sqrt{\pi}\left[\phi\left(X_{1}\right)-\phi\left(X_{2}\right)\right]}
$$

At this stage, for the sake of simplicity I choose $V(x)$ in the form of white-noise: $\overline{V\left(x_{1}\right) V\left(x_{2}\right)}=n_{\mathrm{i}} u^{2} \delta\left(x_{1}-x_{2}\right)$, where $n_{\mathrm{i}}$ is the concentration of "impurities" and $u$ is the "impurity strength". The effective elastic mean free path $\ell$ (in the absence of the interactions) can then be defined as $1 / \ell=n_{\mathrm{i}} u^{2} / a^{2} \omega_{\mathrm{F}}^{2}$, where $\omega_{\mathrm{F}}$ is the (non-universal) ultraviolet energy cut-off (of the order of the Fermi energy). Although in the real GaAs system the impurity potential is long-ranged, the expectation is that this simplification cannot significantly affect the final results, provided that $\ell$ is replaced by the correct mean free path for a more realistic disorder potential [17]. Thus I find the correction to the non-local conductivity:

$$
\begin{aligned}
-\overline{\delta \sigma_{\omega}\left(x, x^{\prime}\right)} & =\frac{2 i e^{2} \bar{\omega}^{2} \omega_{\mathrm{F}}^{2}}{\pi \ell \omega} \int_{-L / 2}^{L / 2} d \bar{x} G_{\bar{\omega}}^{0}(x, \bar{x}) G_{\bar{\omega}}^{0}\left(\bar{x}, x^{\prime}\right) \\
& \times\left.\left[F_{0}(\bar{x})-F_{\bar{\omega}}(\bar{x})\right]\right|_{\bar{\omega} \rightarrow i \omega-\epsilon},
\end{aligned}
$$


where $G_{\bar{\omega}}^{0}$ is the propagator in the absence of disorder and $F_{\bar{\omega}}(x)$ is the $\tau$-Fourier transform of the (inhomogeneous) $2 k_{F}$ density-density correlation function

$$
\begin{gathered}
F(x, \tau) \equiv\langle Q(x \tau, x 0)\rangle_{0} \\
=\exp \left[-\frac{4 \pi}{\beta} \sum_{\bar{\omega}}\left(1-e^{-i \bar{\omega} \tau}\right) G_{\bar{\omega}}^{0}(x, x)\right] .
\end{gathered}
$$

The propagator $G_{\bar{\omega}}^{0}\left(x, x^{\prime}\right)$ satisfies the equation

$$
\left\{\frac{\bar{\omega}^{2}}{v(x) K(x)}-\partial_{x}\left(\frac{v(x)}{K(x)} \partial_{x}\right)\right\} G_{\bar{\omega}}^{0}\left(x, x^{\prime}\right)=\delta\left(x-x^{\prime}\right)
$$

and the following boundary conditions: (i) that $G_{\bar{\omega}}^{0}\left(x, x^{\prime}\right)$ be continuous at $x= \pm L / 2$ and $x=x^{\prime}$; (ii) that $\frac{v(x)}{K(x)} \partial_{x} G_{\bar{\omega}}^{0}\left(x, x^{\prime}\right)$ be continuous at $x= \pm L / 2$; but (iii) be discontinous at $x=x^{\prime}$ so that

$$
-\left.\frac{v(x)}{K(x)} \partial_{x} G_{\bar{\omega}}^{0}\left(x, x^{\prime}\right)\right|_{x=x^{\prime}-0} ^{x=x^{\prime}+0}=1
$$

In addition, I assume that infinitesimal dissipation is present in the leads, so that $G_{\bar{\omega}}^{0}\left( \pm \infty, x^{\prime}\right)=0$. To evaluate the function $F$ in Eq. (9), one needs to know $G_{\bar{\omega}}^{0}\left(x, x^{\prime}\right)$ only for $-L / 2 \leq x=x^{\prime} \leq L / 2$. Straightforward, albeit lengthy, algebra leads to the result

$$
G_{\bar{\omega}}^{0}=\frac{K_{\mathrm{W}}}{2|\bar{\omega}|}+\frac{K_{\mathrm{W}}}{|\bar{\omega}|} \frac{\kappa_{-}^{2} e^{-L / L_{\bar{\omega}}}+\kappa_{+} \kappa_{-} \cosh \left(2 x / L_{\bar{\omega}}\right)}{e^{L / L_{\bar{\omega}}} \kappa_{+}^{2}-e^{-L / L_{\bar{\omega}}} \kappa_{-}^{2}},
$$

where $L_{\bar{\omega}} \equiv v_{\mathrm{W}} /|\bar{\omega}|, v_{\mathrm{W}}$ is the density-wave velocity in the wire and $\kappa_{ \pm} \equiv 1 / K_{\mathrm{W}} \pm 1 / K_{\mathrm{L}}$. I now consider separately the cases of "high" $\left(v_{\mathrm{W}} / L \ll T \ll \omega_{\mathrm{F}}\right)$ and "low" $\left(T \ll v_{\mathrm{W}} / L\right)$ temperatures. (The quotations marks are intended to imply to that the "low" temperature case can be alternatively viewed as the "long" length case and vice versa.)

At "high" temperatures, the second term in Eq. (12) is exponentially small $(\propto \exp (-(L-$ $\left.2 x) / L_{\bar{\omega}}\right)$ unless $\bar{\omega}=0$. The term $\bar{\omega}=0$ gives zero contribution to the sum in Eq. (9), however, as can be seen by performing the infrared regularization of the propagator $(|\bar{\omega}| \rightarrow$ $\sqrt{|\bar{\omega}|^{2}+m^{2}}$ ) and then letting $m \rightarrow 0$. Thus, only the first term in Eq. (12) has to be taken into account. This term is precisely the same as in the case of a homogeneous Luttinger liquid with parameter $K_{\mathrm{W}}$. Already at this stage it can be anticipated that the $T$-scaling of 
the conductance is determined by $K_{\mathrm{W}}$. The function $F$ is now $x$-independent and is given by

$$
F=\left[\frac{2 \pi / \omega_{F} \beta}{\sin \pi \tau / \beta}\right]^{2 K_{\mathrm{W}}}
$$

After the analytic continuation $\bar{\omega} \rightarrow i \omega-\epsilon$, the limit $\omega \rightarrow 0$ can be taken. In this limit the remaining two propagators in Eq. (8) are $x$ - and $x^{\prime}$-independent: $\lim _{\omega \rightarrow 0} G_{\omega}^{0}\left(x, x^{\prime}\right)=i K_{\mathrm{L}} / 2 \omega$ [10]. The rest of the calculations proceeds exactly as in the homogeneous case. The resulting non-local de conductivity is also $x$ - and $x^{\prime}$-independent from which, with the help of Eq. (11), one finds that the dc conductance is $g=\sigma_{0}[20,10]$. Recalling the result for the conductance of a perfect wire, $g_{0}=K_{\mathrm{L}} e^{2} / h$ [9, 10], and restoring $\hbar$, the final result for the conductance can be written as

$$
\bar{g}=g_{0}+\overline{\delta g}=K_{\mathrm{L}} \frac{e^{2}}{h}-C K_{\mathrm{L}}^{2} \frac{L}{\ell}\left[\frac{2 \pi T}{\hbar \omega_{\mathrm{F}}}\right]^{2\left(1-K_{\mathrm{W}}\right)},
$$

where

$$
C=8 \sqrt{\pi} \sin \left(\pi K_{\mathrm{W}}\right) \frac{\Gamma\left(1-K_{\mathrm{W}}\right)}{\Gamma\left(\frac{1}{2}+K_{\mathrm{W}}\right)}\left(\Gamma\left(K_{\mathrm{W}}\right)\right)^{2}
$$

Note that $K_{\mathrm{L}}$ enters only in the prefactors, whereas the exponent of the $T$-scaling is determined by $K_{\mathrm{W}}$. Tracing back through the calculations, we can now see the reason for this. The $K_{\mathrm{L}}$-dependence comes from the propagators in the prefactor of Eq. (8), which depend on the frequency of the applied field $\omega$. In the limit $\omega \rightarrow 0$, they become long-ranged and contain only $K_{\mathrm{L}}$ but not $K_{\mathrm{W}}$. The $T$-scaling comes the propagator entering the function $F$ [Eq. (9)]. This propagator does not depend on $\omega$. In the " high" temperature limit, it becomes short-ranged, and contains only $K_{\mathrm{W}}$ but not $K_{\mathrm{L}}$.

I now turn to the case of "low" temperatures. In previous work dealing with the homogeneous model [2,4, 5], this case was treated by employing the Lorentzian invariance of the $(1+1)$ D field theory which ultimately reduces to a simple recipe: to obtain the result at "low" temperatures, one can take the result for "high" temperatures and replace $T$ by $v / L$, and vice versa. This recipe works, however, only if $L$ has a meaning of the total system size. 
In our inhomogeneous case, $L$ is the length of the wire which is only the part of the system, the total system including both the wire and the leads. Thus, the $T \Leftrightarrow v / L$ equivalence cannot a priori be expected to work. In fact, it will be shown below that such an equivalence exists only if electrons in the leads do not interact, i.e., $K_{\mathrm{L}}=1$.

At "low" temperatures, the sum over $\bar{\omega}$ in Eq. (9) can be replaced by the integral. Performing the integration, I find

$$
\begin{gathered}
F(x, \tau)=\frac{1}{\left[1+\left(\tau \omega_{\mathrm{F}}\right)^{2}\right]^{K_{\mathrm{W}}}} \times \\
\exp \left(-K_{\mathrm{W}} z_{0}\left[\Phi\left(\frac{\tau v_{\mathrm{W}}}{2 L}, 0, z_{0}\right)+z_{0} \Phi\left(\frac{\tau v_{\mathrm{W}}}{2 L}, \frac{L+2 x}{2 L}, z_{0}\right)\right]\right)
\end{gathered}
$$

where $z_{0} \equiv \kappa_{-} / \kappa_{+}$, and

$$
\Phi(x, y, z) \equiv \sum_{n=0}^{\infty} z^{2 n} \ln \frac{x^{2}+(n+1+y)^{2}}{(n+1+y)^{2}}
$$

Evaluation of the Fourier integral $\int d \tau\left(1-e^{i \bar{\omega} \tau}\right) F(x, \tau)$ for arbitrary $\bar{\omega}$ is impossible in this case. As $\bar{\omega}$ has now become a continuous variable $(\beta \rightarrow \infty)$, however, it is possible to find the asymptotics of the integrand of Eq. (8) for small $\bar{\omega}$ and then analytically continue to $i \omega$. Analysis shows that in the limit $\bar{\omega} \rightarrow 0$ the main contribution to the Fourier integral comes from the interval $\tau \geq L / v_{\mathrm{W}}$, where the function $\Phi$ [Eq. (17)] can be replaced by its large- $x$ asymptotic form: $\Phi(x, y, z) \approx \ln x^{2} /\left(1-z^{2}\right)$. It can also be shown that the imaginary part of $F_{0}-F_{\bar{\omega}}$ is asymptotically larger than its real part in the $\bar{\omega} \rightarrow 0$ limit. After simple manipulations one then obtains $F_{0}-F_{\bar{\omega}}=\left(\left(L / L_{\bar{\omega}}\right)^{\alpha} / i \bar{\omega}\right) \times I(\lambda)$, where $\lambda \equiv \omega_{\mathrm{F}} / \bar{\omega}$,

$$
I(\lambda) \equiv \int_{0}^{\infty} d z \frac{z^{-\alpha} \sin z}{\left[1+\lambda^{2} z^{2}\right]^{K_{\mathrm{W}}}}
$$

and $\alpha \equiv 2 K_{\mathrm{L}}\left(K_{\mathrm{L}}-K_{\mathrm{W}}\right)$. As we see, the exponent $\alpha$, which is going to determine the $L$-scaling of $\overline{\delta g}$, depends both on $K_{\mathrm{L}}$ and $K_{\mathrm{W}}$ and is equal to the exponent of the $T$-scaling [i.e, to $2\left(1-K_{\mathrm{W}}\right)$ ] only if $K_{\mathrm{L}}=1$. Thus, the equivalence of $T$ - and $L$-scalings exists only if the electrons in the leads do not interact. To be consistent with the physical content of the model, I will now consider only the case $K_{\mathrm{L}}=1$. We now need to evaluate the asymptotic 
behavior of the integral (18) for $\lambda \rightarrow \infty$. This can be done by using the Parseval formula for Mellin transforms [21], according to which one has

$$
I(\lambda)=\int_{c+i \infty}^{c+i \infty} \frac{d s}{2 \pi i} \lambda^{-s} \Gamma(s / 2) \Gamma(\bar{s}) \sin (\pi \bar{s} / 2)
$$

where $\bar{s} \equiv 2 K_{\mathrm{W}}-1-s$, and $c$ is chosen in the domain of analyticity of the integrand $\left(0 \leq \operatorname{Re} s \leq 2 K_{\mathrm{W}}\right)$. For $\lambda \rightarrow \infty$, the leading contribution to the sum over residues is given by the smallest second-order pole at $s=2 K_{\mathrm{W}}$. I then find

$$
\left.I(\lambda)\right|_{\lambda \rightarrow \infty} \approx \Gamma\left(K_{\mathrm{W}}\right) \frac{\ln \lambda}{\lambda^{2 K_{\mathrm{W}}}}
$$

In the limit being considered, $F_{0}-F_{\bar{\omega}}$ is $x$-independent, and the integration over $\bar{x}$ in Eq. (8) again gives $L$. The continuation $\bar{\omega} \rightarrow i \omega-\epsilon$ can now be performed. Separating the real and imaginary parts of $\ln \lambda$ in Eq. (20), I finally obtain the real part of the conductance in the form

$$
\overline{\operatorname{Re} g}=\frac{e^{2}}{h}-\frac{e^{2}}{h} 2 \pi^{2} \Gamma\left(K_{\mathrm{W}}\right) \frac{L}{\ell}\left[\frac{2 L \hbar \omega_{\mathrm{F}}}{v_{\mathrm{W}}}\right]^{2\left(1-K_{\mathrm{W}}\right)}
$$

Similar analysis can be performed in the case of a single potential barrier inserted into the Luttinger-liquid wire which, in the case of a uniform interaction strength, was considered first in Refs. [3: 1 ]. The result is identical to the case of the extended random potential considered above: in both the weak and strong disorder cases the $T$-and $L$-dependences of the conductance are determined by the parameter $K_{\mathrm{W}}$ of the wire.

The generalization for case of electrons with spin can readily be performed. As in the homogeneous case [四, 5], the exponent $2-2 K_{\mathrm{W}}$ is replaced by $2-K_{\mathrm{W}}^{\rho}-K_{\mathrm{W}}^{\sigma}$, or, if the $S U(2)$ symmetry of the underlying Hubbard model is preserved [i.e., $K_{\mathrm{W}}^{\sigma}=1$ ] by $1-K_{\mathrm{W}}^{\rho}$, where $K_{\mathrm{W}}^{\rho / \sigma}$ are the parameters of the charge/spin parts of the Luttinger-liquid in the wire.

This work was supported by the NSF under grant DMR89-20538. I am grateful to P. Goldbart, E. Fradkin, D. Loss, N. Sandler, and M. Stone for stimulating discussions. P. Goldbart, N. Sandler, and M. Stone have also read the manuscript and made valuable comments. P. Goldbart deserves special thanks for attracting my attention to and providing me with a copy of Ref. [21]. 


\section{REFERENCES}

[1] F. D. M. Haldane, J. Phys. C 14, 2585 (1981).

[2] W. Apel and T. M. Rice, Phys. Rev. B 26, 7063 (1982).

[3] C. L. Kane and M. P. A. Fisher, Phys. Rev. Lett. 68, 1220 (1992).

[4] C. L. Kane and M. P. A. Fisher, Phys. Rev. B 46, 15233 (1992).

[5] M. Ogata and H. Fukuyama, Phys. Rev. Lett. 73, 468 (1994).

[6] A. Furusaki and N. Nagaosa, Phys. Rev. B 47, 4631 (1993).

[7] K. A. Matveev, D. Yue, and L. I. Glazman, Phys. Rev. Lett. 71, 3351 (1993); Phys. Rev. B 49, 1966 (1994).

[8] S. Tarucha, T. Honda, T. Saku, Sol. State Commun. 94, 413 (1995).

[9] I. Safi and H. J. Schulz, cond-mat/9505079 (unpublished).

[10] D. L. Maslov and M. Stone, cond-mat/9505098 (unpublished).

[11] K. A. Matveev and L. I. Glazman, Physica B 71, 266 (1993).

[12] Y. Imry, in Directions in Condensed Matter Physics, edited by G. Grinstein and G. Mazenko (World Scientific, Singapore, 1986), Vol. 1, p. 101.

[13] R. Landauer, Z. Phys. B 68, 217 (1987).

[14] L. I. Glazman, G. B. Lesovik, D. E. Khmel'nitskii and R. I. Shekhter, Pis'ma Zh. Eksp. Teor. Fiz. 48, 218 (1988) [Sov. Phys. JETP. Lett. 48, 238 (1988)].

[15] Y. B. Levinson, Zh. Eksp. Teor. Fiz. 95, 2175 (1989) [Sov. Phys. JETP. 68, 1257 (1989)].

[16] This assumption is adequate for the experimental situation studied in Ref. [8], where $L$ was at least $\simeq 6$ smaller than the elastic mean free path of the unbounded 2DEG and the total observed change in the conductance was $1-5 \%$ of $e^{2} / h$ depending on the wire 
length.

[17] See L. I. Glazman and M. Jonson, Phys. Rev. B 44, 3810 (1991) for the calculation of the effective mean free path for a realistic disorder potential.

[18] R. Shankar, Int. J. Mod. Phys. B 4, 2371 (1990).

[19] A. Luther and I. Peschel, Phys. Rev. Lett. 32, 992 (1974).

[20] H. J. Schulz, 1994 Les Houches lecture notes (cond-mat/9503150).

[21] N. Bleistein and R. Handelsman, Asymptotic Expansion of Integrals (Dover, NY, 1986). 